\title{
Langmuir
}

This document is confidential and is proprietary to the American Chemical Society and its authors. Do not copy or disclose without written permission. If you have received this item in error, notify the sender and delete all copies.

\section{Capillary penetration into inclined circular glass tubes}

\begin{tabular}{|r|l|}
\hline Journal: & Langmuir \\
\hline Manuscript ID & la-2015-03904x.R1 \\
\hline Manuscript Type: & Article \\
\hline Complete List of Authors: & $\begin{array}{l}\text { Trabi, Christophe; Nottingham Trent University, School of Science and } \\
\text { Technology } \\
\text { Ouali, F. Fouzia; Nottingham Trent University, School of Science and } \\
\text { Technology } \\
\text { McHale, Glen; Northumbria University, School of Computing, Engineering \& } \\
\text { Information Sciences } \\
\text { Javed, Haadi; Nottingham Trent University, School of Science and } \\
\text { Technology } \\
\text { Morris, Robert; Nottingham Trent University, School of Science and } \\
\text { Technology } \\
\text { Newton, Michael; The Nottingham Trent University, School of Science and } \\
\text { Technology }\end{array}$ \\
\hline
\end{tabular}

SCHOLARONE ${ }^{\text {m }}$

Manuscripts 


\title{
Capillary penetration into inclined circular glass
}

\author{
tubes \\ Christophe L. Trabi, F. Fouzia Ouali", Glen McHale "\#, Haadi Javed, Robert H. Morris and \\ Michael I. Newton \\ School of Science and Technology, Nottingham Trent University, \\ Clifton Lane, Nottingham NG11 8NS, UK. \\ ${ }^{\#}$ Faculty of Engineering \& Environment, Northumbria University, Ellison Place, Newcastle upon \\ Tyne NE1 8ST, UK \\ *Corresponding Authors: \\ Fouzia.ouali@ntu.ac.uk; Tel: +44 0 (115) 83490, \\ glen.mchale@,northumbria.ac.uk; Tel: +44 (0)191 2273660
}




\begin{abstract}
The spontaneous penetration of a wetting liquid into a vertical tube against the force of gravity and the imbibition of the same liquid into a horizontal tube (or channel) are both driven by capillary forces and described by the same fundamental equations. However, there have been few experimental studies of the transition from one orientation to the other. We report systematic measurements of capillary penetration of polydimethylsiloxane oils of viscosities 9.6, 19.2 and $48.0 \mathrm{mPas}$ into glass capillary tubes. We first report the effect of tube radii $R$ between $140 \mu \mathrm{m}$ and $675 \mu \mathrm{m}$ on the dynamics of spontaneous imbibition We show that the data can be fitted using the exact numerical solution to the governing equations and that these are similar to fits using the analytical visco-gravitational approximation. However, larger diameter tubes show a rate of penetration slower than expected using an equilibrium contact angle and the known value of liquid viscosity. To account for the slowness, an increase in viscosity by a factor $(\eta / \rho)_{\text {scaling }}$ is needed. We show full agreement with theory requires the ratio $R / \kappa^{-1} \sim 0.1$ or less, where $\kappa^{-1}$ is the capillary length. In addition, we propose an experimental method that enables the determination of the dynamic contact angle during imbibition, which gives values that agree with the literature values. We then report measurements of dynamic penetration into the tubes of $R=190 \mu \mathrm{m}$ and $650 \mu \mathrm{m}$ for a range of inclination angles to the horizontal, $\varphi$, from $5^{\circ}$ to $90^{\circ}$. We show that capillary penetration can still be fitted using the visco-gravitational solution, rather than the Bosanquet solution which describes imbibition without gravity, even for inclination angles as low as $10^{\circ}$. Moreover, at these low angles, the effect of the tube radius is found to diminish and this appears to relate to an effective capillary length, $\kappa^{-1}(\varphi)=\sqrt{\gamma_{L V} / \rho g \sin \varphi}$.
\end{abstract}


Keywords: Lucas-Washburn, Bosanquet, capillary imbibition, microfluidic channel, dynamic contact angle.

Supporting Information: Larger versions of the diagrams. This information is available free of charge via the internet at http://pubs.acs.org

\section{INTRODUCTION}

The flow of small amounts of liquid along microfluidic channels is generally governed by capillarity-driven imbibition. Capillary imbibition has already been studied extensively in printing, water infiltration in porous material, lab-on-chip and many other applications ${ }^{1-5}$. However, many previous investigations are based on theories focusing on two specific cases: horizontally oriented capillaries, where gravity can be ignored, and vertically oriented capillaries, where gravity has a major influence. The theory underlying these two cases, and the more general case of a capillary oriented at an arbitrary angle, $\varphi$, to the horizontal, is described by a differential equation, which involves inertial forces, viscous forces, hydrostatic pressure and the capillary forces (see e.g. Ouali et al. ${ }^{6}$ ). Depending on the relative strength of these forces the rise, or penetration length, of the liquid scales differently with time. There are a number of known analytical solutions to approximations of the governing differential equation. These include the early stage inertia dominated phase ${ }^{7}$, the late stage Lucas-Washburn viscous regime neglecting gravity $^{8-9}$, the more general Bosanquet solution which incorporates both of these solutions, but neglects gravity ${ }^{10}$, and the visco-gravitational solution ${ }^{9,11}$. The latter solution describes capillaries in which the inertial force is neglected and the capillary force is balanced by the viscous force and gravity and this regime is usually assumed to occur after the Lucas-Washburm regime ${ }^{12-15}$. 
In liquids where the viscous forces are small (low viscosity liquids and wider tubes), the capillary force is balanced by inertia and gravity, and imbibition is characterised by the inertial regime at the very early stages, followed by a viscosity damped oscillatory regime about the equilibrium height, which completely replaces the Lucas-Washburn regime ${ }^{16-19}$. Recently, Das and Mitra ${ }^{18}$ found that the different regimes of capillary rise in vertical capillaries are determined by the ratio of the Ohnesorge number $(O h)$ to the Bond number $(B o)$. The limit $O h / B o \gg 1$ corresponds to a monotonic rise dominated by the Lucas-Washburn regime, followed by gravitational effects at the end of the rise. $O h / B o \ll 1$ corresponds to the case when the rise is characterised by the damped oscillatory behaviour and there is no Lucas-Washburn regime. When $O h / B o \sim 1$, an intermediate regime is observed. In recent work, we developed a numerical procedure for fitting experimental data to the exact differential equation in which the viscous force dominates inertia and reported analytical expressions for the transition between the Bosanquet and visco-gravitational solutions ${ }^{6}$. In that work our experimental focus was on capillary rise in channels of rectangular cross section where one side of the channel was open to air; this was motivated by an interest in how the process of capillary rise crosses over into microfluidics with open and closed channels.

An interesting issue for capillary driven imbibition into near horizontal microfluidic channels is the extent to which gravity can be neglected. In this work we report a systematic investigation into capillary driven penetration of polydimethylsiloxane (PDMS) oils into circular cross-section glass tubes at angles of orientation from the vertical to near horizontal (around $5^{\circ}$ from the horizontal). Our experimental work covers a range from one clearly described by a viscogravitational solution to a near horizontal tube where it might be expected a Bosanquet equation would be the most appropriate description. In section 2, we first provide a summary of the 
theory, including the governing differential equation, and the visco-gravitational and Bosanquet solution, in a form that includes the effects on gravitational forces from the angle of inclination to the horizontal, $\varphi$. We also summarize numerical results detailing the cross-over from the Bosanquet to the visco-gravitational solution as the most accurate approximation to the governing differential equation. In sections 3 and 4, we consider the effect of tube diameter on the fitting of capillary rise in vertically oriented tubes.

In our previous work, we noted that although fits to the exact equations were possible using an effective viscosity, numerical agreement with known values of viscosity only occurred for small diameter tubes. This was probably due to the effective viscosity compensating for the use of a fixed value of contact angle rather than including a time dependent early stage dynamic contact angle ${ }^{20,6}$. Here, we conclude that this effect is controlled by $R / \kappa^{-1}(\varphi)$, where $\kappa^{-1}(\varphi)$ is the capillary length taking into account the reduction in gravitational force due to the angle of inclination, and becomes less of an issue as a tube approaches the horizontal orientation. Our results of capillary penetration into tilted tubes also inform understanding of the transition from a vertical orientation towards a horizontal orientation thereby investigating a progressively decreasing influence of gravity. We find that even for very low angles of $10^{\circ}$ the PDMS oils we investigated obey a visco-gravitational solution. We also report a new experimental method that enable the determination of the velocity dependent dynamic contact angle that gives values in agreement with the literature.

\section{THEORETICAL APPROACH}

The theory of capillary-driven imbibition of a liquid of viscosity, $\eta$, and density, $\rho$, into a circular cross-section tube of radius $R$, at an arbitrary angle to the horizontal, $\varphi$, results in a 
governing equation for the penetration length along the tilted tube, $x(t)$, as a function of time, $t$,

of,

$\frac{1}{2} \frac{d}{d t}\left(\frac{d x^{2}}{d t}\right)=b-g \sin \varphi x-a x\left(\frac{d x}{d t}\right)$

where $g=9.81 \mathrm{~m} \mathrm{~s}^{-2}$ is the acceleration due to gravity. In this form of the equation, the viscous coefficient $a$ has dimensions of inverse time $\left(\mathrm{s}^{-1}\right)$ and the capillary coefficient term $b$ has dimensions of (speed $)^{2}\left(\mathrm{~m}^{2} \mathrm{~s}^{-2}\right)$, and are given by,

$$
a=8 \eta / \rho R^{2} \quad \text { and } \quad b(t)=2 \gamma_{L V} \cos \theta(t) / \rho R
$$

The force driving the imbibition (or rise) is capillarity and the forces resisting the imbibition are gravity and viscous forces. If a velocity dependent, and hence time dependent, dynamic contact angle, $\theta=\theta(t)$, is assumed a further defining equation relating the dynamic contact angle to the instantaneous speed and physical parameters is required ${ }^{14,20-22}$. However, it is often assumed that the contact angle can be approximated to a constant $\theta=\theta_{A}$ (the advancing contact angle) and with this assumption eq. (1) can be fitted numerically to a set of experimental data ${ }^{6}$. There are also several approximations to eq. (1) with $\theta=\theta_{A}$ that allow analytical solutions in the cases where gravity is dominant or where gravity can be neglected; for a recent summary see Ouali et $\mathrm{al}^{6}$.

When $g \sin \varphi \rightarrow 0$ so that the capillary is horizontally oriented, the solution to eq. (1) is the Bosanquet solution $^{10}$,

$$
x^{2}(t)=\left(\frac{2 b}{a^{2}}\right)\{a t-[1-\exp (-a t)]\}
$$

This solution includes the long time limit solution $x(t)=(2 b / a)^{1 / 2} t^{1 / 2}$, which is the LucasWashburn solution when the viscous term dominates, and also the short time limit solution, 
$x(t)=b^{1 / 2} t$, which is the inertial solution of Quéré ${ }^{7}$. As shown by Washburn ${ }^{9}$, and discussed by Fries and Dreyer ${ }^{11,17,24}$ (see also Mumley et al., ${ }^{12}$ and Krotov and Rusanov ${ }^{13}$ ), eq. (1) also has a visco-gravitational solution,

$$
t=-\frac{a b}{(g \sin \varphi)^{2}}\left(\frac{x}{x_{e}}+\ln \left(1-\frac{x}{x_{e}}\right)\right)=-\frac{a x_{e}^{2}}{b}\left(\frac{x}{x_{e}}+\ln \left(1-\frac{x}{x_{e}}\right)\right)
$$

where the inertial term has been ignored; a constant contact angle and $x=0$ at $t=0$ have also been assumed in deriving this solution. The equilibrium penetration length is given by,

$$
x_{e}=\frac{b}{g \sin \varphi}
$$

The fact eq. (4) is an analytical solution with time as a function of position, which cannot be easily inverted, does not prevent fitting of experimental data since time can be fitted as a function of measured position as easily as position as a function of measured time. Interestingly, the extreme long time limit of eq. (4) is an exponential approach to equilibrium, $x / x_{e} \rightarrow(1-\exp (-$ $\mathrm{t} / \tau)$ ) with a time constant $\tau=a b /(g \sin \varphi)^{2}$. The short time limit with $x / x_{e}<<1$ is the LucasWashburn solution $x(t)^{2}=(2 b / a) t$, so that the short time limit of the visco-gravitational solution corresponds to the long-time limit of the Bosanquet equation; these solutions should therefore have a cross-over.

\subsection{Identifying regimes of approximation}

It is useful to separate an experimental data series into a form where each range of approximation from inertial to Lucas-Washburn to visco-gravitational can be easily identified. Motivated by the series expansion of the logarithmic term in the visco-gravitational solution, we consider the dependence of $t / x^{2}$ as a function of $x$. In this form, the small time limit (inertial approximation) of the Bosanquet solution eq. (3) is, 


$$
\frac{t}{x^{2}} \approx \frac{1}{\sqrt{b} x}=\frac{1}{\sqrt{x_{e} g \sin \varphi} x}
$$

As time increases this inertial solution crosses over to the Bosanquet solution (eq. (3)), which in the long time limit (Lucas-Washburn) approximation is dominated by the linear term in $t$ and so gives,

$$
\frac{t}{x^{2}} \rightarrow \frac{a}{2 b}
$$

Equation (7) also matches the small time approximation of the visco-gravitational solution,

$$
\frac{t}{x^{2}} \rightarrow\left(\frac{a}{2 b}\right)\left[1+\frac{2}{3}\left(\frac{x}{x_{e}}\right)+\frac{1}{2}\left(\frac{x}{x_{e}}\right)^{2}+\ldots\right]
$$

At much longer times $t / x^{2}$ logarithmically diverges as a function of $x$ because $x$ approaches $x_{e}$ exponentially.

The changeover of behaviour from a $1 / x$ to a constant of $a / 2 b$ to a divergence should be visually distinctive and enable the appropriate fitting equation (eq. (3) or eq. (4) or the inertial or Lucas-Washburn approximations) to be used. The inertial to Lucas-Washburn transition occurs when $x$ satisfies both eq. (6) and eq. (7), i.e.

$$
x_{I \rightarrow L W} \sim \frac{2 \sqrt{b}}{a} \propto \frac{\sqrt{R^{3}}}{\eta}
$$

which is equivalent to $t_{I \rightarrow L W}=2 / a$, so $x_{I \rightarrow L W}$ increases with $R$. The Lucas-Washburn to viscogravitational transition occurs when the first order correction in eq. (8) becomes non-negligible, $2 x / 3 x_{e} \sim 0.1$, i.e.

$$
x_{L W \rightarrow V G} \sim 0.15 x_{e}
$$




\subsection{Cross-over between Bosanquet and visco-gravitational solutions}

Numerically the initial penetration length is best described by the Bosanquet solution (eq. (3)) until it crosses over with the visco-gravitational solution (eq. (4)). Above this cross-over the visco-gravitational solution ever more closely agrees with the exact numerical solution as the capillary penetration length tends to its equilibrium value; however, it always lies above the exact numerical solution. The cross-over time, $t_{c}$, can be calculated numerically as a function of $g \sin \varphi$. as shown in Ouali ${ }^{6}$, and this gives,

$$
t_{c} \approx \frac{1}{f\left(a t_{c}\right)}\left(\frac{9 x_{e}^{2}}{8 a b}\right)^{1 / 3}
$$

where

$$
f\left(a t_{c}\right)=\frac{1-\left(1-\exp \left(-a t_{c}\right)\right) /\left(a t_{c}\right)}{\left(1-\exp \left(-a t_{c}\right)\right)^{2 / 3}}
$$

In the limit of large $a t_{c}$ the function $f\left(a t_{c}\right) \rightarrow 1$, and hence eq. (11), gives the cross-over time as a $2 / 3^{\text {rd }}$ power law in the equilibrium penetration length, i.e. $t_{c} \propto x_{e}{ }^{2 / 3}$. The cross-over length, $x_{c}$, as a fraction of the equilibrium length, $x_{e}$, can be written using the equilibrium length ${ }^{6}$,

$$
\frac{x_{c}}{x_{e}} \approx\left(\frac{3 b}{a^{2} x_{e}^{2}}\right)^{1 / 3}
$$

\subsection{Non-dimensional analysis of the different regimes}

To identify the different regimes, the relationship between $t / x^{2}$ and $x$ can be used, and in order to generalize the obtained solutions the data can be plotted in a non-dimensional form as $T / X^{2}$ against $X=x / x_{\mathrm{e}}$, where $T=t / \tau$ and $\tau$ is a characteristic time motivated by the visco-gravitational solution (eq. (4)) and defined as

$$
\tau=\left(a b / g^{2} \sin ^{2} \varphi\right)=a x_{e} / g \sin \varphi
$$


In this form, the first of the limiting regimes, in increasing value of $x$, is the inertial regime, which from eq. (6) is,

$$
\left(\frac{T}{X^{2}}\right)_{I}=\frac{t x_{e}^{2}}{\tau x^{2}}=\frac{1}{2}\left\{1-\frac{1}{a \tau T}(1-\exp (-a \tau T))\right\}^{-1} \approx\left(\frac{1}{\sqrt{a \tau}}\right) \frac{1}{X}
$$

This crosses over into the Bosanquet solution (eq. (3)). The second regime, the Lucas-Washburn solution, is given from eq. (7) as,

$$
\left(\frac{T}{X^{2}}\right)_{L W}=\frac{t x_{e}^{2}}{\tau x^{2}}=\frac{1}{2}
$$

and the third regime, the visco-gravitational solution, is given from eq. (4) as,

$$
\left(\frac{T}{X^{2}}\right)_{\mathrm{VG}}=\frac{t x_{e}^{2}}{\tau x^{2}}=-\left(\frac{1}{X}\right)\left[1+\left(\frac{1}{X}\right) \ln (1-X)\right]
$$

In this form, the cross-over, $X_{c}=x_{c} / x_{e}$, from Bosanquet to visco-gravitational is given from eq. (13) by,

$$
X_{c}=\left(\frac{3}{a \tau}\right)^{1 / 3}
$$

and the defining differential equation is,

$$
\frac{1}{2 a \tau} \frac{d}{d T}\left(\frac{d X^{2}}{d T}\right)=1-X-X\left(\frac{d X}{d T}\right)
$$

From eq. (9) and eq. (18), the cross-over from the inertial to Lucas-Washburn regime would be expected at,

$$
\begin{aligned}
& X_{I \rightarrow L W}=\frac{x_{I \rightarrow L W}}{x_{e}} \sim \frac{2}{\sqrt{a \tau}}=\frac{2 X_{c}^{3 / 2}}{\sqrt{3}} \\
& X_{I \rightarrow L W}<X_{c} \text { for } X_{\mathrm{c}}<0.75 \text {. This was found to be the case in all our experiments. }
\end{aligned}
$$




\section{EXPERIMENTAL METHODS}

To observe experimentally the effect of a diminishing influence of gravity, an experimental set-up was developed to allow the monitoring of capillary penetration into a tilted circular crosssection tube (fig. 1). Due to difficulties in liquid penetration at the entry to almost horizontal tubes, the angular range used was $5^{\circ}$ to $90^{\circ}$, where $90^{\circ}$ gives a vertically mounted tube. Data for penetration length and time taken for tubes at different tilt angles for tubes can then be numerically fitted by eq. (1) and compared to fits to the analytical solutions for the two regimes of zero gravity (Bosanquet solution, eq. (3)) or the visco-gravitational regime (eq. (4)). The effectiveness of the fits can then be compared to the expected form based on the cross-over time.

One difficulty with this approach, as described in our previous work ${ }^{6}$, is that although the numerical fitting of eq. (1) to experimental data is possible using a constant contact angle, it necessarily requires the $a$ parameter to be numerically adjusted (via an effective viscosity significantly larger than known value) to obtain fits that agree with the experimental data. This was interpreted as due to a dynamic contact angle that was much larger during the initial stages of capillary rise, thus reducing the initial capillary pull compared to expectations based on a quasi-static equilibrium contact angle. A large effective viscosity then compensates for the constant contact angle assumption since it reduces the rate of imbibition in the earlier stages of capillary rise. The validity of this interpretation will be discussed in section 4.3.

To look at the need for an effective viscosity, prior to taking systematic data for tilt angles, we considered the effect of tube diameter on the extent to which capillary rise and penetration data can be fitted by eq. (1) with known values of viscosity. Capillary rise of polydimethylsiloxane (PDMS) oil (Dow Corning, Xiameter PMX-200) of viscosity $\eta=9.6,19.2$ and $48.0 \mathrm{mPas}( \pm 5 \%)$ at $25^{\circ}$, with corresponding densities of $\rho=913,930$ and $950 \mathrm{~kg} \mathrm{~m}^{-3}$ and surface tension $\gamma_{\mathrm{LV}}=19.8$ 
$\mathrm{mN} \mathrm{m}^{-1}$, into vertically oriented tubes of radius $140 \mu \mathrm{m}, 185 \mu \mathrm{m}, 295 \mu \mathrm{m}, 430 \mu \mathrm{m}, 525 \mu \mathrm{m}, 575 \mu \mathrm{m}$ and $675 \mu \mathrm{m}( \pm 5 \mu \mathrm{m})$ were examined. This range was chosen because the capillary length is $\kappa^{-}$ ${ }^{1}=\left(\gamma_{L V} \rho g\right)^{1 / 2}=(1.47 \pm 0.02) \mathrm{mm}$ and this gives a tube radius to capillary radius range of $R / \kappa^{-1} \approx 0.1$ to 0.5 .

Fig. 1 shows the experimental set up. The PDMS oil reservoir was raised into contact with the capillary tube. Recording was made via a CCD camera (up to 60 frames per second) and the evolution of the meniscus penetration length along the tube, $x$, recorded as a function of time, $t$; reference graticules were used alongside each tube for calibration of length (fig. 1). Using our image analysis approach led to large data sets for each individual experiment and so data points presented in the results section are representative ones from reduced data sets. Experiments at each viscosity and for each tube were repeated five times to establish reproducibility of the data. The ambient temperature was recorded and the value was stable for a given experiment and varied in the range $23-26{ }^{\circ} \mathrm{C}$ between experiments. The variation in viscosity from the temperature variation was confirmed using rheology measurements to be less than $5 \%$ of the values at $25^{\circ} \mathrm{C}$.

In the experiments using tilted tubes, two radii of $190 \mu \mathrm{m}$ and $650 \mu \mathrm{m}$ were considered with the tube orientation systematically changed in steps of $10^{\circ}$ from a completely vertical orientation to $10^{\circ}$ from the horizontal; a final set of capillary imbibition measurements at an angle of inclination of $5^{\circ}$ from the horizontal was also used. In these experiments the CCD camera was also orientated at the same angle so that the tube remained aligned vertical in the field of view thereby enabling the measurement of the oil penetration length along the titled tube. Video capture commenced before the reservoir was brought into contact with the tube and continued until either the liquid achieved an equilibrium length or, for shallow title angles of the thinner 
tube, had reached the maximum field of view. For the majority of cases, the full penetration to an equilibrium length along the tilted tube could be observed. In all cases, the fitting of eq. (1) to the data was possible. After each experiment the video of liquid penetrating into the tube was loaded into image processing software, the calibration factor for length determined, a zero time assigned to the frame immediately before the liquid started to penetrate the tube, the final frame subtracted from all preceding frames and a threshold image processing operation performed. These videos were then loaded into MATLAB ${ }^{\circledR}$ (Mathworks) and the position of the meniscus in every frame was calculated as a function of time.

The variation of capillary rise with time (penetration length in tilted tubes) data were fitted to the governing differential equation, eq. (1), and to the visco-gravitational solution, eq. (4), using Mathematica ${ }^{\circledR}$ with $a$ and $b$ as free fitting parameters. We also numerically determined the solution to eq. (1) using i) the theoretical value of $a$ (determined by the literature reference value of viscosity) and a $b$ value scaled to match the observed rise (equivalent to scaling the surface tension/density) and ii) the values of parameters from the fitting to the visco-gravitational solution, to visually verify the extent to which the solutions were in agreement.

\section{RESULTS AND DISCUSSIONS}

\subsection{Vertical rise and effect of tube diameter}

We observed that the plot of capillary rise with time followed the expected shape from the numerical solution to the governing differential equation (eq. (1)), or equivalently the viscogravitational solution (eq. (4)). The equilibrium length tended to be slightly different to that predicted using the literature reference parameter values, but this could be corrected by using a scaling factor $(\sim 1)$ for $b$ (or equivalently $\left(\gamma_{L V} / \rho\right)_{\text {scaling }}$ ). However, as previously reported ${ }^{6}$, for large radii tubes the data appeared to follow curves with much higher viscosity (or more 
correctly higher $(\eta / \rho)$ or equivalently higher values of $a$, and, in agreement with other reports in the literature ${ }^{18,25}$. An example of this is given by the dashed line in the inset to fig. $2(R=430 \mu \mathrm{m}$, $\eta=9.6 \mathrm{mPas}, \rho=913 \mathrm{~kg} \mathrm{~m}^{-3}$ and $\gamma_{\mathrm{LV}}=19.8 \mathrm{mN} \mathrm{m}^{-1}$ with $\left(\gamma_{L V} / \rho\right)_{\text {scaling }}=0.9651$ to match the equilibrium length).

To fit the experimental data, an increase in effective viscosity of $a_{\text {scaling }}=(\eta / \rho)_{\text {scaling }}=2.628$ is required. In this case, and all other viscosities and radii examined for vertical tubes, the viscogravitational solution could be fitted to the data. Moreover, using the same parameters gave a numerical solution to eq. (1) which on a penetration length-time plot was indistinguishable to the visco-gravitational solution. We observed that the scaling factor required to fit the data, reduced towards unity as tubes of smaller radii were used. The main part of fig. 2 summarizes the scaling factors $a_{\text {scaling }}=(\eta / \rho)_{\text {scaling }}$ as a function of the ratio of tube radius to capillary length, $R / \kappa^{-1}$. Scaling factors required to fit the data for capillary rise of PDMS oils of viscosities $9.6 \mathrm{mPas}$ $(\diamond \diamond \diamond), 19.2 \mathrm{mPas}($ ooo) and $48.0 \mathrm{mPas}(\Delta \Delta \Delta)$ inside glass. The data and theory come into agreement when $R / \kappa^{-1}<0.1$. The dashed lines are to guide the eye for the trend with the ratio of radius to capillary length. It is notable that the curves in fig. 2 do not follow a steadily increasing or decreasing trend with viscosity and do not scale with viscosity in a sample manner.

We observed visually that as the PDMS penetrates into a tube the contact angle appears to evolve from a high angle value towards $0^{\circ}$. In the first frames, the meniscus is flat but then shapes itself toward a spherical form as the liquid rises into the tube. When the equilibrium is reached, the observed contact angle is close to zero degrees. This appears to confirm the interpretation of Ouali ${ }^{6}$ and others ${ }^{14,25,26}$ that eq. (4) using a constant value of $\theta=0^{\circ}$ is incorrect and this is found to have a measurable effect on the rate of imbibition in the early stage of penetration as discussed in section 4.3. Figure 2 confirms that this effect systematically reduces 
for thin capillary tubes, $R<<\kappa^{-1}$, in which the surface area to volume ratio increases as the tube radius decreases. Recently, Das and Mitra $^{18}$ attributed the increase of the scaling factor to a nonparabolic fluid velocity profile, characterized by a steeper velocity gradient of the liquid near the wall of the tube compared to the bulk, giving rise to an increased viscous force (hence of $a$ ). The difference between gradients near the wall and bulk reduces as the radii of the channels decreases, thus, improving agreement with theory in thinner tubes. This interpretation may be consistent with a dependence of the effective viscosity on the ratio $R / \kappa^{-1}$. However, irrespective of the physical reason it appears experimentally that this is a key parameter combination determining whether an effective viscosity is required when assuming a constant contact angle.

\subsection{Vertical rise and different regimes}

Figs. $3 \mathrm{a}$ and $3 \mathrm{~b}$ show the rise dynamics of PDMS oil in the form of a semi-log plot of $X=x / x_{e}$ as a function of time, for three data sets in the smallest vertical tube $(R=140 \mu \mathrm{m})$ at $\eta=19.2 \mathrm{mPas}$ and the widest $(R=675 \mu \mathrm{m})$ at $\eta=9.6 \mathrm{mPas}$ as respectively. In each graph, the corresponding Bosanquet and visco-gravitational fits are shown as a solid and dashed lines respectively, and the vertical dotted lines show cross-over penetration lengths $X_{I-L W}$ and $X_{\mathrm{c}}$. The graphs show that the visco-gravitational solution is the best analytical fit to the rise for most of the range.

To analyze the data for capillary rise, the rise versus time to the equilibrium length, $x_{e}$ of the three data set (eg. figs $3 \mathrm{a}$ and $3 \mathrm{~b}$ ) were used as the starting points to plot the data sets using $T / X^{2}=(t / \tau) \times x_{e}^{2} / x^{2}$ against $X$ to overlay the data as $X \rightarrow 1$ where the characteristic time, $\tau$, (eq. (14)) is a fitting parameter. Examples of the results are shown in fig. $3 \mathrm{c}$ and $3 \mathrm{~d}$. In the early stages of capillary rise, there is a small offset in time due to the frame rate used, for the data moves the value of $T / X^{2}$, but the data points tend to lie on a similar curve. However, the associated errors 
due to the frame rate seem to have a sizeable effect at the very early stages. In the final part of the capillary rise the fit to the data is very sensitive to choice of the $\tau$ parameter.

In the smallest radius $(R=140 \mu \mathrm{m})$ vertical tubes at $\eta=19.2 \mathrm{mPas}$ (fig $3 \mathrm{c}$ ), we found the $x_{e}$ value on one data set needed to be adjusted by $0.5 \%$. A value of $\tau=29 \mathrm{~s}$ was chosen to fit the visco-gravitational solution (eq. (16) (dashed line); this also determined the Lucas-Washburn solution, eq. (16) (horizontal dashed line). Finally, a value of $a=\tau g \sin \varphi / x_{\mathrm{e}}=9680 \mathrm{~s}^{-1}\left(\varphi=90^{\circ}\right)$ (eq. (14)) was used for the Bosanquet solution (eq. (3) or eq. (15). The values of $a$ and $\tau$ then give the Bosanquet to visco-gravitational cross-over, $X_{\mathrm{c}}=0.022$ (eq. (18)) and the cross-over penetration length $X_{I-L W}=0.004$ (eq. (20) (also shown as the vertical dotted lines in fig. 3b). It is of note that the value of $a=9680 \mathrm{~s}^{-1}$ obtained from this method agrees well with the corresponding average value $a=9652 \mathrm{~s}^{-1}$ (SD $178 \mathrm{~s}^{-1}$ ) obtained from the analytical and numerical fits of penetration versus time data in fig $3 \mathrm{a}$.

The widest tube (fig. $3 \mathrm{~d}$ ), corresponding to $R=675 \mu \mathrm{m}$ and $\eta=9.6 \mathrm{mPas}$, gives a theoretical value of $X_{\mathrm{c}}=0.509$, thus $X_{I \rightarrow L W}=0.419$, but this is larger than the cross over from Lucas-Washburn to visco-gravitational regimes expected at $X_{L W \rightarrow V G} \sim 0.15$. Thus the Lucas-Washburn regime should not occur for this data set. So at no time could the Bosanquet solution be regarded in the long time limit and nor can the visco-gravitational solution be regarded in the short time limit. However, since capillary penetration is observed to be slower than theoretically predicted (fig. 2), using the larger fitted value for the effective viscosity in eq. (18) would give a smaller effective value $X_{I \rightarrow L W}=0.074$ and a corresponding $X_{\mathrm{c}}=0.160$ which is close to $X_{L W \rightarrow V G} \sim 0.150$ as shown by the vertical dotted lines in fig. $3 \mathrm{~d}$ (and the horizontal line in fig. 3b). This suggests, for these tubes, a regime where the transition to the Lucas-Washburn regime occurs just as gravity 
starts to play a role. However, the visco-gravitational solution should still be the best fit to the data for most of the range $\left(X>X_{\mathrm{c}}=0.16\right)$ as is clearly shown in fig. $3 \mathrm{~b}$.

We note that, there is a kink at intermediate stages in the region of $x / x_{\mathrm{e}} \sim 0.8$ in fig. $3 \mathrm{~d}$. $O h / B o=$ $\frac{\eta}{g} \sqrt{\frac{\gamma_{L V}}{\rho^{3} R^{5}}} \sim 2$ for these tubes, which may suggest a behavior that is intermediate between oscillatory and viscous driven flow as proposed by Das and Mitra ${ }^{18}$ or a transition to the oscillatory regime in these wide tubes for $\eta=9.6 \mathrm{mPas}$. Further investigations would be required to verify this. Furthermore, we note that a similar transitional behavior was also observed in vertical tubes of radii $R=525 \mu \mathrm{m}$ and $575 \mu \mathrm{m}$ for $\eta=9.6 \mathrm{mPas}$, but that the Lucas-Washburn regime was found to occur in the smaller tubes $(R=140 \mu \mathrm{m}, 185 \mu \mathrm{m}, 295 \mu \mathrm{m}$ and $430 \mu \mathrm{m})$ for $\eta=9.6 \mathrm{mPas}$ and in all vertical tubes (including $R=675 \mu \mathrm{m}$ ) for $\eta=19.2 \mathrm{mPas}$ and $48.0 \mathrm{mPas}$.

The data in figs. $3 \mathrm{c}$ and $3 \mathrm{~d}$ deviate from the visco-gravitational solution in the early to intermediate stages and implies that the data never reaches a situation when the Lucas-Washburn regime actually applies even though the flow is viscous driven. A similar behavior was observed in all other tubes and viscosities and could not be accounted for in terms of experimental errors, such as those associated with the frame rate. A possible reason for this systematic deviation will be discussed next.

\subsection{The effect of a dynamic contact angle}

The above analysis assumes a constant static contact angle value $\theta=0^{\circ}$, which is not consistent with the experimental observations at the very early stages of rise and with other work in the literature $^{14,25,26}$. Next, we attempt to account for the deviation of the data from the viscogravitational solution in the early to intermediate stages in figs. $3 \mathrm{c}$ and $3 \mathrm{~d}$ in terms of a velocity dependent dynamic contact angle $\theta(t)$. 
Since accurate measurements of the $\theta(t)$ were not possible in this work, we have estimated $\theta(t)$ by fitting Hoffman's empirical measurements of the variation of $\theta(t)$ with capillary number $C a=V \eta / \gamma_{\mathrm{LV}}$ (where $V$ is the liquid front velocity) in the region $10^{-5} \leq C a \leq 10^{-2}$ obtained for silicone oils in glass tubes ${ }^{22}$ and used by many authors (e.g. Katoh et al. ${ }^{26}$ ). We find that the dynamic contact angle can be expressed as $\theta(t)=\theta+228 \times C a^{0.3287}$, where $\theta(t)$ and $\theta$ are in degrees (referred to hereafter as the Hoffman correlation). We note that there are alternative numerical correlations that could be used for $\theta(t)$ (see, e.g., Bracke et al. ${ }^{23}$,Fries \& Dreyer $^{24}$;Chebbi ${ }^{28}$ ). Figs $4 \mathrm{a}$ and $4 \mathrm{~b}$ show the values of $\theta(t)$ for the data of fig $3 \mathrm{a}$ and $3 \mathrm{~b}$ respectively estimated using the above Hoffman correlation.

Given that $x_{\mathrm{e}}$ and $\tau$ (eq. 14) are both proportional to $b$, and, if we now consider that the contact angle is $\theta(t)$, then $b(t) \propto \cos \theta(t)$, so the vertical axis $T / X^{2}=\left(t / x^{2}\right) \times\left(x_{e}^{2} / \tau\right) \propto b(t) \propto \cos \theta(t)$. So, for each experimental data point in figs. $3 \mathrm{c}$ and $3 \mathrm{~d}$, using the static angle value $\theta$ overestimates the scaling factor of $T / X^{2}$ by a factor $\cos \theta / \cos \theta(t)=1 / \cos \theta(t)$ (as $\theta=0^{\circ}$ ) compared to the viscogravitational solution which uses a constant value $\theta$. So, in order to be able to compare the scaled experimental data $T / X^{2}$ in figs. 3c and $3 \mathrm{~d}$ to the visco-gravitational solution, $T / X^{2}$ must be multiplied by the corresponding $\cos \theta(t)(\theta(t)$ from figs. $4 \mathrm{a}$ and $4 \mathrm{~b})$. Figs. $4 \mathrm{c}$ and $4 \mathrm{~d}$ show that a much improved agreement with the visco-gravitational solution (eq. (17)), including at the very early stages of rise, is obtained as a result.

Similar results were observed for all other tube radii and viscosities indicating that a dynamic contact angle needs be taken into account in any quantitative analysis at the early stages of imbibition in agreement with other work in the literature ${ }^{22}$. It is of note, however, that the use of the dynamic contact angle only slightly affects the fitted value of $\tau$, hence $a$, by $\leq 5 \%$. This 
indicates that: a) it cannot account solely for the increase in the scaled viscosity with radius as we proposed previously ${ }^{6}$, and b) that use of a static angle in the numerical and analytical fits should still be adequate to describe imbibition. However, the method above is solely experimental and does not attempt to include the effects of $\theta(t)$ in the governing equations or in the numerical fits.

\subsection{A new method for estimating the dynamic contact angle}

The results of figs. $4 \mathrm{c}$ and $4 \mathrm{~d}$ also indicate that it may be possible to estimate the dynamic contact angle from the penetration length along the tube-time data for any given tube in viscous dominated flow by the following new procedure: i) fit the penetration length vs time data either using the visco-gravitational solution or numerical solution to determine $a$ and $x_{\mathrm{e}}$, ii) rescale the data to obtain $T / X^{2}=\left(\left(t / x^{2}\right) \times x_{e}^{2} / \tau\right)_{\text {scaled }}$ where $\tau$ is calculated from the fitted values using eq. (14), and iii) for each experimental point $X=x / x_{\mathrm{e}}, \theta(t)$ can be estimated from $\cos \theta(t)=\left(\frac{T}{X^{2}}\right)_{V G} /\left(\frac{T}{X^{2}}\right)$, where $\left(\frac{T}{X^{2}}\right)_{V G}$ is given by eq. (17). Figure 5 shows the obtained estimates of $\theta(t)$, shown as filled-in circles, using this method for $R=140 \mu \mathrm{m}$ and $\eta=19.2 \mathrm{mPas}$ (data of fig. 3c). Also shown are the values of contact angle, shown as unfilled squares, obtained using the Hoffman correlation (fig. 4a). As anticipated, an excellent agreement is observed between the two independent methods of estimating $\theta(t)$ for the data used. Similar results were obtained for other tubes and viscosities.

In addition, the values of $\theta(t)$ estimated using the method above agree very well with the recently proposed empirical trend which relates $x / x_{\mathrm{e}}$, and experimentally measured $\theta(t)$ for vertical and tilted geometries as $\frac{x}{x_{e}}=A \exp \left(\frac{-(\theta(t)-B)^{2}}{2 C^{2}}\right)^{1 / 4 R}\left(\right.$ Heshmati and Piri $\left.{ }^{25}\right)$, where $R$ is 
the radius of the tube in $\mathrm{mm}$, as shown by the solid line in fig. 5 . The solid line uses the same values of $A=1.03, B=-2.47$ and $C=27.7$ as Heshmati and Piri. We found that the Heshmati and Piri formula fits data for all tubes and inclinations, although a slightly lower value for $C$ was sometimes needed for better fits. This agreement gives us confidence in our proposed methodology of estimating $\theta(t)$.

\subsection{Different regimes of flow in tilted tubes}

Fig. 6 shows the effect of tilt angle $\varphi\left(5 \leq \varphi \leq 90^{\circ}\right)$ on the fitted equilibrium penetration length $x_{\mathrm{e}}$ for tubes of radii $650 \mu \mathrm{m}$ and $190 \mu \mathrm{m}$. The dashed lines show the corresponding theoretical predictions for $x_{\mathrm{e}}$. A good agreement with predictions is obtained (to within 5\%) apart from the shallowest tubes $\left(\varphi=5\right.$ and $\left.10^{\circ}\right)$ where differences of up to $15 \%$ were observed between fitted values and theory in the thin tube. These larger differences are attributed to the difficulty in observing the meniscus at the very early stages in these almost horizontal tubes because of the external meniscus covering the tube and because of larger uncertainties in determining the value of $x_{\mathrm{e}}$ where penetration lengths are beyond the maximum field of view of the camera.

Capillary penetration for different $\varphi$ were also fitted using the Bosanquet, numerical and visco-gravitational solutions in tubes of radii $190 \mu \mathrm{m}$ and $650 \mu \mathrm{m}$ assuming a constant advancing contact angle $\theta=0^{\circ}$. Figs $7 \mathrm{a}$ and $7 \mathrm{~b}$ show the penetration dynamics for oil viscosity $9.6 \mathrm{mPas}$ in tubes of radii $650 \mu \mathrm{m}$ and $190 \mu \mathrm{m}$ respectively and the dashed curves show the corresponding visco-gravitational fits. Here again the visco-gravitational was found to be adequate to fit the data.

We next discuss the different regimes of flow in tilted tubes and the role of gravity. $X_{c} \sim\left(3 b / a^{2} x_{e}^{2}\right)^{1 / 3}$ (eq. (13)) and $X_{I \rightarrow L W} \sim 2 X_{c}^{3 / 2} / \sqrt{3}$ (eq. (20), so can be expressed in terms of 
$R$ and $\varphi$ as $X_{c} \sim\left(R^{5} g^{2} \sin ^{2} \varphi / \eta^{2}\right)^{1 / 3}$ and $X_{I \rightarrow L W} \propto\left(R^{5} g^{2} \sin ^{2} \varphi / \eta^{2}\right)^{1 / 2}$. The largest possible value $X_{\mathrm{c}}$ is, thus, for the most vertical $\left(\varphi=90^{\circ}\right)$ and widest tubes $(R=650 \mu \mathrm{m})$ at $\eta=9.6 \mathrm{mPas}$ for which an effective value of $X_{\mathrm{c}}=0.123$ was needed to fit the data, and $X_{\mathrm{c}}$ decreases to 0.077 and 0.048 for $\eta=19.2 \mathrm{mPas}$ and $48.0 \mathrm{mPas}$ respectively. Moreover, for a given tube radius $R$ and viscosity, $X_{\mathrm{c}}$ should decrease with decreasing $\varphi$, hence with decreasing gravitational force, although we note that the corresponding penetration length values $x_{c} \propto\left(R^{2} / g \sin \varphi \eta^{2}\right)^{1 / 3}$ and $x_{e} \propto 1 /(\operatorname{Rg} \sin \varphi)$ both increase with decreasing $\varphi$. Our analysis found the effective values of $X_{\mathrm{c}}$ to decrease with decreasing $\varphi$, but more slowly than predicted theoretically. We attribute this slower decrease to the dependence of the effective viscosity on $\varphi$ discussed below in section 4.5.

In addition, we found that for both $R=650 \mu \mathrm{m}$ and $R=190 \mu \mathrm{m}$, for any given viscosity and tilt angles, liquid imbibition is best fitted by the visco-gravitational, rather than the Bosanquet solution, for most of the range, even for angles as low as $10^{\circ}$ as shown in fig. 8 . A value $X_{\mathrm{c}}=0.031\left(x_{\mathrm{c}}=1.32 \mathrm{~mm}, x_{e}=42.00 \mathrm{~mm}\right)$ was obtained for $R=650 \mu \mathrm{m}$ and $\eta=48.0 \mathrm{mPas}$ at $\varphi=10^{\circ}$. This indicates that gravity needs to be taken into account (for $X>X_{c}$ ) even in these near horizontal tubes and that ignoring gravity, as is often the case in microfluidic devices ${ }^{27}$, theoretically overestimates the rate of imbibition. This is particularly important in narrow capillaries and micro-sized channels since $X_{\mathrm{c}}$ reduces with $R\left(X_{c} \propto R^{5 / 3}\right)$ which may have implications in understanding the flow dynamics, design and operation of microfluidic systems based on capillary filling and flow control ${ }^{5,29}$. The above analysis may also be of interest in capillary flow under micro-gravity conditions as it may help explain the different regimes of flow reported in the literature ${ }^{30,31}$. 


\subsection{Tilted tubes and the effective capillary length}

The capillary length characterizing the balance between surface tension and gravitational forces is given by $\kappa^{-1}=\left(\gamma_{\mathrm{LV}} / \rho g\right)^{1 / 2}$. Using the gravitational force along the tube for an inclined tube gives

$$
\kappa^{-1}(\varphi)=\sqrt{\frac{\gamma_{L V}}{\rho g \sin \varphi}} .
$$

As the angle $\varphi$ decreases, the capillary length $\kappa^{-1}(\varphi)$ increases which in turn means that the ratio of the radius over the capillary length decreases. For our vertically oriented tubes of radii $140 \mu \mathrm{m}$ to $675 \mu \mathrm{m}$ the ratio of radius to capillary length is $R / \kappa^{-1}\left(90^{\circ}\right)=0.095$ to 0.458 , whereas for the two tilted tubes of radii $190 \mu \mathrm{m}$ and $650 \mu \mathrm{m}$, the ratios are $0.129 \sin ^{1 / 2} \varphi$ and $0.442 \sin ^{1 / 2} \varphi$. For the lowest tilt angle of $5^{\circ}$ these give 0.038 and 0.13 , respectively.

Imbibition in tilted tubes was also found to be slower than predicted and an increase in effective viscosity of $a_{\text {scaling }}=(\eta / \rho)_{\text {scaling }}$ was also needed to fit the data, and that, for a given viscosity and tube radius $(R=190 \mu \mathrm{m}$ or $650 \mu \mathrm{m}),(\eta / \rho)_{\text {scaling }}$ reduces with reducing tilt angle $\varphi$. This suggests that the effect of tube radius reduces with decreasing gravitational force (via decreasing tilt angles) which appears to relate to the effective value of $R / \kappa^{-1}(\varphi)$. Fig. 9 shows that $(\eta / \rho)_{\text {scaling }}$ increases with $R / \kappa^{-1}(\varphi)$, with good agreement between theory and experiment for values of $R / \kappa^{-1}(\varphi)<0.1$, and that the trend is consistent with the results in vertical tubes discussed above (also included in fig. 9 for comparison). These observations show that the effect of the radius becomes less of an issue in horizontal capillaries and micro-channels, and may, therefore, have important implications in our understanding of the dynamics of flow in such systems. It would be very interesting to repeat the measurements with different liquids to establish whether 
the trend observed in fig. 9 holds, and whether the magnitude of the viscosity scaling factor $a_{\text {scaling }}$ is also dependent on the liquid-solid interface as suggested in some reports in the literature $^{28,32}$.

\section{CONCLUSIONS}

In this work, we have presented a systematic study of the dynamics of capillary penetration of PDMS oils in vertical and tilted glass tubes at a range of angles of inclination to the horizontal, $\varphi$ $\left(10^{\circ} \leq \varphi \leq 90\right)$. The penetration length versus time data were fitted using the exact numerical solution and the approximate analytical visco-gravitational and Bosanquet solutions using a constant contact angle. We found the visco-gravitational solution to be the most appropriate analytical approximation to the exact solution even for lower inclinations (around $10^{\circ}$ ) when one might expect the Bosanquet solution to be the best approximation for most of the range. This indicates that gravity may need to be taken into consideration in any quantitative analysis of rate of imbibition, especially in micro and nano-sized channels. From the fits, we found that the theory agrees with experiment in small tube radii, $R$, when $R / \kappa^{-1}(\varphi)<0.1$, where $\kappa^{-1}(\varphi)=\sqrt{\gamma_{L V} / \rho g \sin \varphi}$ is an effective capillary length which uses the component of acceleration due to gravity along the axis of the tube, $g \sin \varphi$. For tubes of larger radii that satisfy $R / \kappa^{-1}(\varphi)>0.1$, the flow is found to be slower than predicted and showing an increase with $R / \kappa^{-}$ ${ }^{l}(\varphi)$ for all tubes and tilt angles. Moreover, at fixed radii, we observed, for the first time, that effect of the tube radius becomes less of an issue when the gravitational force is reduced at lower tilt angles. 
We also showed that by non-dimensional rescaling and re-plotting the imbibition data, it is, in principle, possible to separate the different regimes of flow. The re-scaling also enabled us to develop a new experimental method that allows the determination of the dynamic contact angle $\theta(t)$ during imbibition. An excellent agreement was obtained between our values of $\theta(t)$ and those given by a recent empirical formula suggested by Heshmati and Piri ${ }^{25}$. Our analysis found the dynamic contact angle to play an important role at the early stages of imbibition, in agreement with other reports in the literature, but that the use of a constant contact angle was still adequate to fit the dynamic data for the later stages. This indicates that the dynamic contact angle cannot solely account for the slower rise than anticipated in the wider tubes. Further measurements will be needed to validate the method further using different channel geometries, liquids and capillaries.

\section{ACKNOWLEDGEMENTS}

The authors acknowledge financial assistance from the U.K. Engineering and Physical Sciences Research Council (Grant No. EP/E063489/1). HJ would like to acknowledge Nottingham Trent University for financial support. We would also like to than Dr David Fairhurst for his help with the viscosity measurements. 


\section{REFERENCES}

(1) Schoelkopf, J.; Gane, P. A. C.; Ridgway, C. J.; Matthews, G. P. Practical observation of deviation from Lucas - Washburn scaling in porous media. Colloids Surf. 2002, 206, 445-454.

(2) Brody, J. P.; Yager, P.; Goldstein, R. E.; Austin, R. H. Biotechnology at low Reynolds numbers. Biophys. J. 1996, 71, 3430-3441 (1996).

(3) Marmur, A; Cohen, R. D. Characterization of porous media by the kinetics of liquid penetration: The vertical capillaries model. J. Colloid Interface Sci. 1997, 189, 299-304.

(4) Shirtcliffe, N. J.; McHale, G.; Newton, M. I.; Pyatt, F. B.; Doerr, S. H. Critical conditions for the wetting of soils. Appl. Phys. Lett. 2006, 89, 094101-094101.

(5) Squires, T.; Quake, S. R. Microfluidics: Fluid physics at the nanoliter scale. Rev. Mod. Phys. 2005, 77, 977-1026.

(6) Ouali, F. F. ; McHale, G.; Javed, H.; Trabi, C.; Shirtcliffe, N. J.; Newton, M. I. Wetting considerations in capillary rise and imbibition in closed square tubes and open rectangular crosssection channels. Microfluid. Nanofluid. 2013, 15, 309-326.

(7) Quéré, D. Inertial capillarity. Europhys. Lett. 1997, 39, 533-538.

(8) Lucas, R. Ueber das Zeitgesetz des Kapillaren Aufstiegs von Flussigkeiten. Kolloid Z. 1918, 23, 15-22.

(9) Washburn, E. W. The dynamics of capillary flow. Phys. Rev. 1921, 17, 273-283.

(10) Bosanquet, C. H On the flow of liquids into capillary tubes. Philos. Mag. Ser. 6 1923, 45, $525-53$. 
(11) Fries, N.; Dreyer, M. An analytic solution of capillary rise restrained by gravity. J. Colloid Interface Sci. 2008, 320, 259-263.

(12) Mumley, T. E.; Radke, C. J.; Williams, M. C. Kinetics of liquid/liquid capillary rise: I. Experimental observations. J. Colloid Interface Sci. 1986, 109, 398-412.

(13) Krotov, V. V.; Rusanov, A. I. Physicochemical hydrodynamics of capillary systems. Imperial College Press: London (ISBN-13: 978-1860941603), 1999, 222.

(14) Hamraoui, A.; Thuresson, K.; Yaminsky, V.; Nylander, T. Can a dynamic contact angle be understood in terms of a friction coefficient? J. Colloid Interface Sci. 2000, 226, 199-204.

(15) Hamraoui, A.; Nylander, T. Analytical approach for the Lucas-Washburn equation. $J$. Colloid Interface Sci. 2002, 250, 415-421.

(16) Quéré, D.; Raphaël, É. Rebounds in a Capillary Tube. Langmuir 1999, 15, 3679-82.

(17) Fries, N.; Dreyer, M. The transition from inertial to viscous flow in capillary rise. $J$. Colloid Interface Sci. 2008, 327, 125-128.

(18) Das, S.; Mitra, S. K. Different regimes in vertical capillary filling. Phys. Rev. E 2013, 87, 063005.

(19) Masoodi, R.; Languri, E.; Ostadhossein A. Dynamics of liquid rise in a vertical capillary tube, J. Colloid Interface Sci. 2013, 389, 268-272.

(20) Popescu, M. N.; Ralston, J. S. R. Capillary rise with velocity-dependent dynamic contact angle. Langmuir 2008, 24, 12710-12716. 
(21) Rose, W.; Heirs, R. W. Moving interfaces and contact angle rate-dependence. J. Colloid Sci. 1962, 17, 39-48.

(22) Hoffman, R. L. A study of the advancing interface. I. Interface shape in liquid-gas systems. J. Colloid Interface Sci. 1975, 250, 228-241.

(23) Bracke, M.; De Voeght,F.; Joos P.The kinetics of wetting: the dynamic contact angle

Prog. Colloid Polym. Sci. 1989, 79, 142-149

(24) Fries, N.; Dreyer, M. Dimensionless scaling methods for capillary rise. J. Colloid Interface Sci. 2009, 338, 514-518.

(25) Heshmati, M.; Piri, M., Experimental investigation of dynamic contact angle and capillary rise in tubes with circular and non-circular cross sections. Langmuir 2014, 30, 14151-14162.

(26) Katoh, K.; Wakimoto, T.; Yamamoto, Y.; Ito. T. Dynamic wetting behaviour of a triplephase contact line in several experimental systems. Exp. Therm. Fluid Sci. 2015, 60, 354-360.

(27) Chebbi R. Dynamics of liquid penetration into capillary tubes. J. Colloid Interface Sci. 2007, 315, 255-260.

(28) Andrukh, T.; Monaenkova, D.; Rubin, B., Lee; W. K.; Kornev , K. G. Meniscus formation in a capillary tube and the role of contact line friction. Soft Matter 2014, 10, 609-615.

(29) Safavieh, R.; Juncker, D. Capillarics: pre-programmed, self-powered microfluidic circuits built from capillary elements. Lab Chip 2013, 21, 4180-89.

(30) Dryer M.; Delagodo A.; Rath H. J. Capillary rise of liquid between parallel plates under micro-gravity. J. Colloid Interface Sci. 1994, 163, 158-168. 
(31) Strange, M.; Dreyer, M.E.; Rath, H. J. Capillary driven flow in circular cylindrical tubes. Phys. Fluids 2003, 15, 2587-2601.

(32) O’Loughlin, M.; Wilk, K.; Priest, C.; Ralston, J.; Popescu, M. N. Capillary rise of aqueous glycerol solutions in glass capillaries: A critical examination of the Washburn equation, $J$. Colloid Interface Sci. 2013, 411, 257-264. 
Figure 1: A schematic of the experimental set up showing the glass capillary tube (a) tilted at an angle $\varphi\left(5^{\circ} \leq \varphi \leq 90^{\circ}\right)$. The liquid in the reservoir (b) was slowly brought up (shown by vertical arrows) until spontaneous filling starts. Recording was made with the CCD camera (c) which is oriented at the same angle $\varphi$ as the sample so that the tube remained vertical to the field of view of the camera, as shown in (d). The evolution of the penetration length along the tubes is recorded as a function of time. 


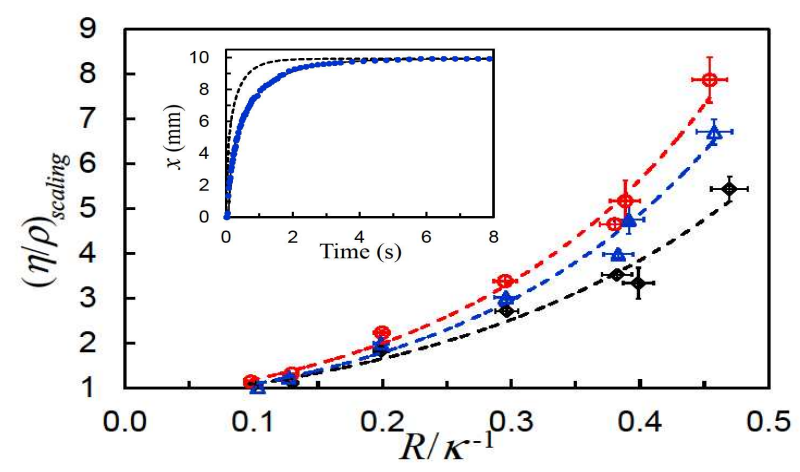

Figure 2. Scaling factors required to fit the data for capillary rise of PDMS oils of viscosities $9.6 \mathrm{mPas}(\diamond \diamond \diamond), 19.2 \mathrm{mPas}($ ooo) and $48 \mathrm{mPas}(\Delta \Delta \Delta)$ inside vertical glass tubes. The dashed lines are to guide the eye for the trend with the ratio of radius to capillary length, $R / \kappa^{-1}$. Inset: Capillary rise of 9.6 mPas PDMS oil inside a tube of radius $R=430 \mu \mathrm{m}$. Solid circles are data points, dashed curve is the numerical solution of eq. (1) using $\rho=913 \mathrm{~kg} \mathrm{~m}^{-3}, \gamma_{L V}=19.8 \mathrm{mN}$ $\mathrm{m}^{-1}, \theta=0^{\circ}$, and solid curve through the data points is visco-gravitational solution with $\eta_{\text {scaling }}=2.628$. Both the dashed and solid curves use $\left(\gamma_{L V} / \rho\right)_{\text {scaling }}=0.965$ to match the observed equilibrium length. Larger versions of the graphs are shown in the supporting information. 

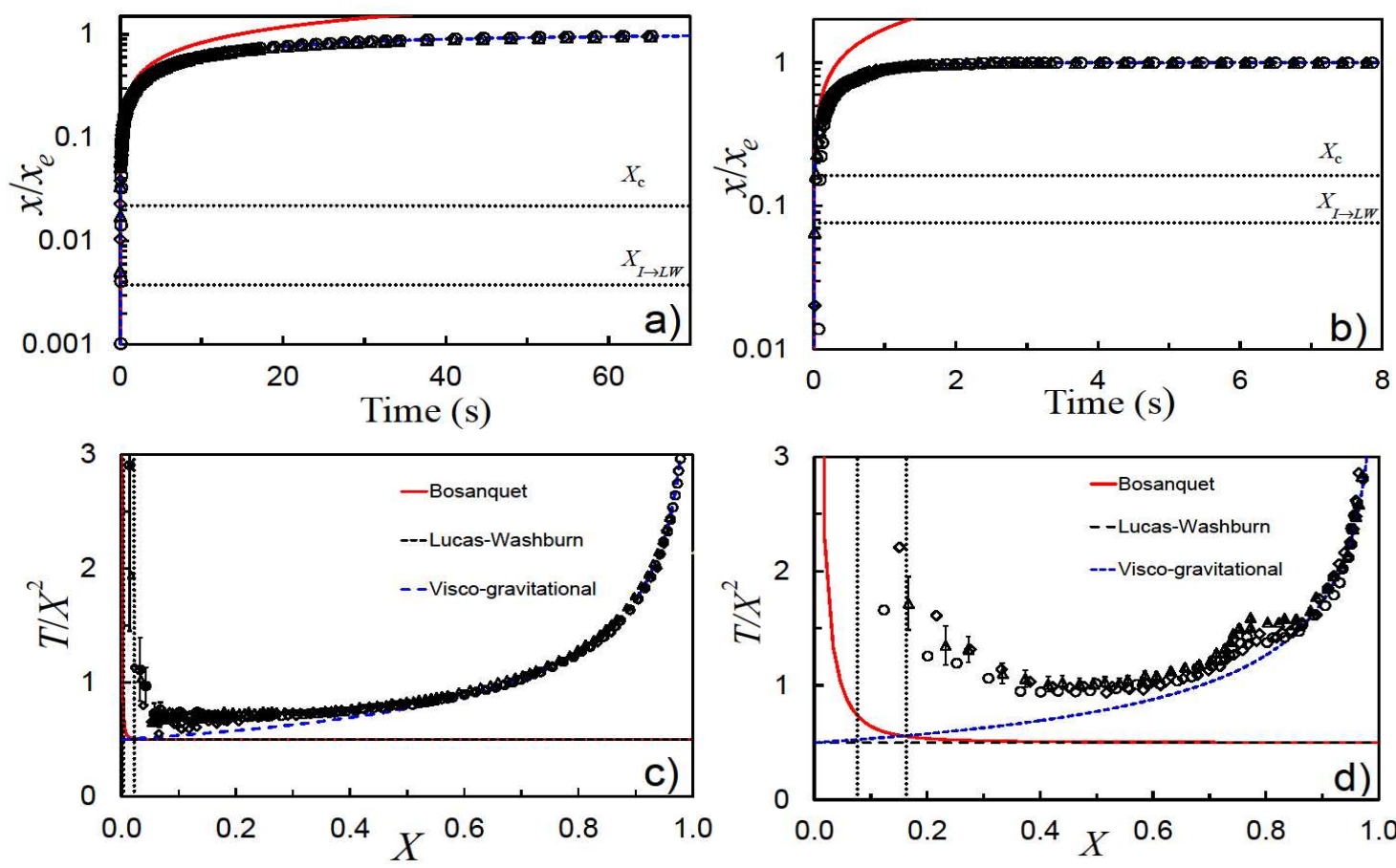

Figure 3. The rise dynamics in the form of a semi-log plot of $X=x / x_{e}$ as a function of time, $t$, for three data sets for a) $R=140 \mu \mathrm{m}$ and $\eta=19.2 \mathrm{mPas}$, and b) $R=675 \mu \mathrm{m}$ and $\eta=9.5 \mathrm{mPas}$. In each graph, the equilibrium lengths, $x_{e}$ have been taken from the Mathematica fits. c) and d) are non-dimensional scaling and re-plotting of the data of a) and b) respectively in the form of $T / X^{2}$ with $X$, where $T=t / \tau$ ( $\tau$ is a characteristic fitting time). In the graphs, the symbols represent the experimental data, the lines represent the Bosanquet solution, eq. (15) (-), the LucasWashburn solution, eq. (16) (- -) and the visco-gravitational fits eq.17 (---). The dotted lines represent the cross-over penetration lengths $X_{I-L W}$ (eq. (20)) and $X_{\mathrm{c}}$ (eq. (18)). In c) $\tau=29 \mathrm{~s}$; $a=\tau g \sin \varphi / x_{\mathrm{e}}=9680 \mathrm{~s}^{-1} ; X_{I \rightarrow L W}=0.0038$ and $X_{c}=0.022$, and in d) $\tau=0.7 \mathrm{~s} ; a=\tau g \sin \varphi / x_{\mathrm{e}}=1045 \mathrm{~s}^{-1}$; $X_{I \rightarrow L W}=0.074$ and $X_{c}=0.16$. 

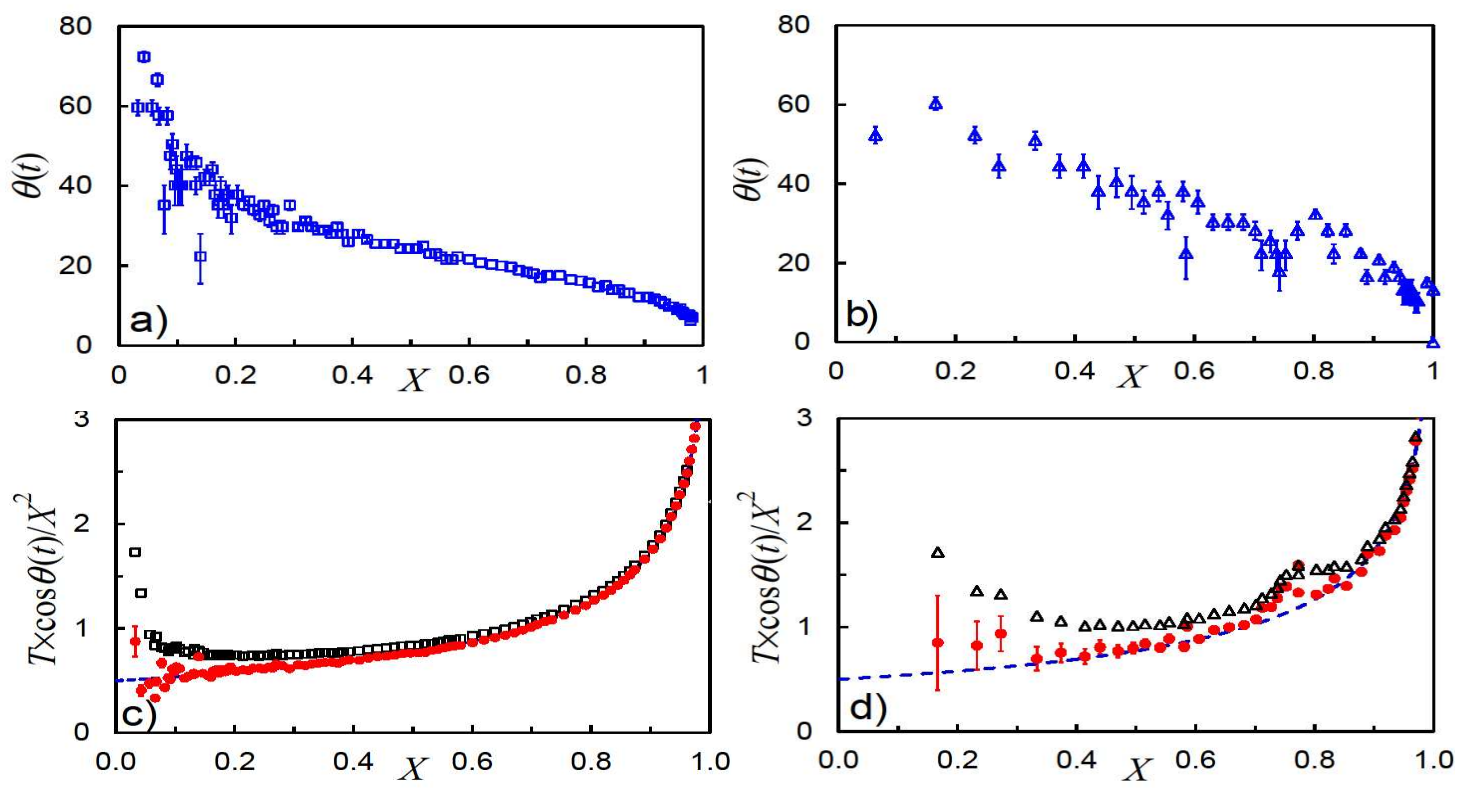

Figure 4: The effect of a dynamic contact angle $\theta(t)$. For each experimental data point in fig $3 \mathrm{a}$ and $3 \mathrm{~b}, \theta(t)$ was estimated using the Hoffman correlation ${ }^{22}$ and the corresponding variation of $\theta(t)$ with $X$ are shown for a) $R=140 \mu \mathrm{m}$ and $\eta=19.2 \mathrm{mPas}$, and b) $R=675 \mu \mathrm{m}$ and $\eta=9.6 \mathrm{mPas}$. c) and d) show the effect of adjusting the scaled data of fig $3 \mathrm{c}$ and $3 \mathrm{~d}$ respectively from $T / X^{2}$ (open symbols) to $T \times \cos \theta(t) / X^{2}$ (closed symbols). $\tau$ needed adjusting from 29 to $28 \mathrm{~s}^{-}$ ${ }^{1}$ in c), and from 0.70 to $0.68 \mathrm{~s}^{-1}$ in d). A much improved agreement with the visco-gravitational solution (dashed line) is observed as result. 


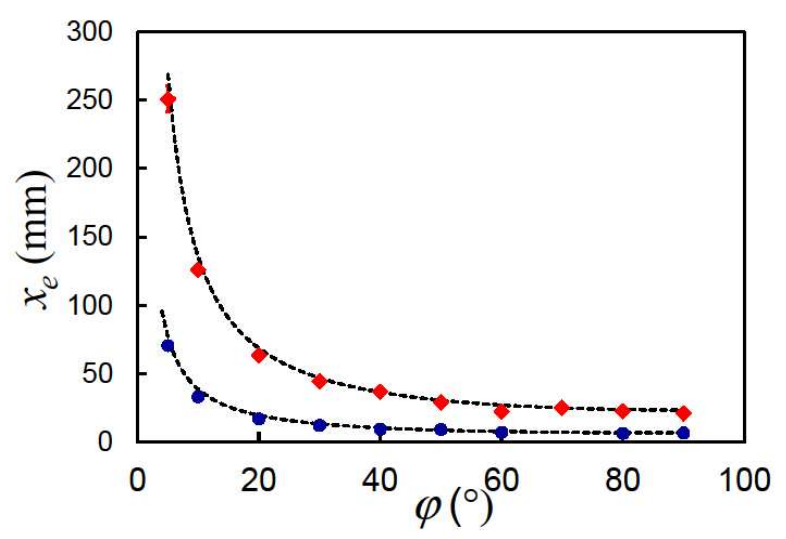

Figure 6: Dependence of equilibrium length of PDMS oils, $x_{\mathrm{e}}$, on tilt angle, $\varphi$, in glass tubes of radii $R=650 \mu \mathrm{m}(\bullet \bullet \bullet)$ and b) $R=190 \mu \mathrm{m}(\bullet \bullet)$. The dashed lines show the theoretical predictions for $x_{\mathrm{e}}$ determined using eq. (5). 

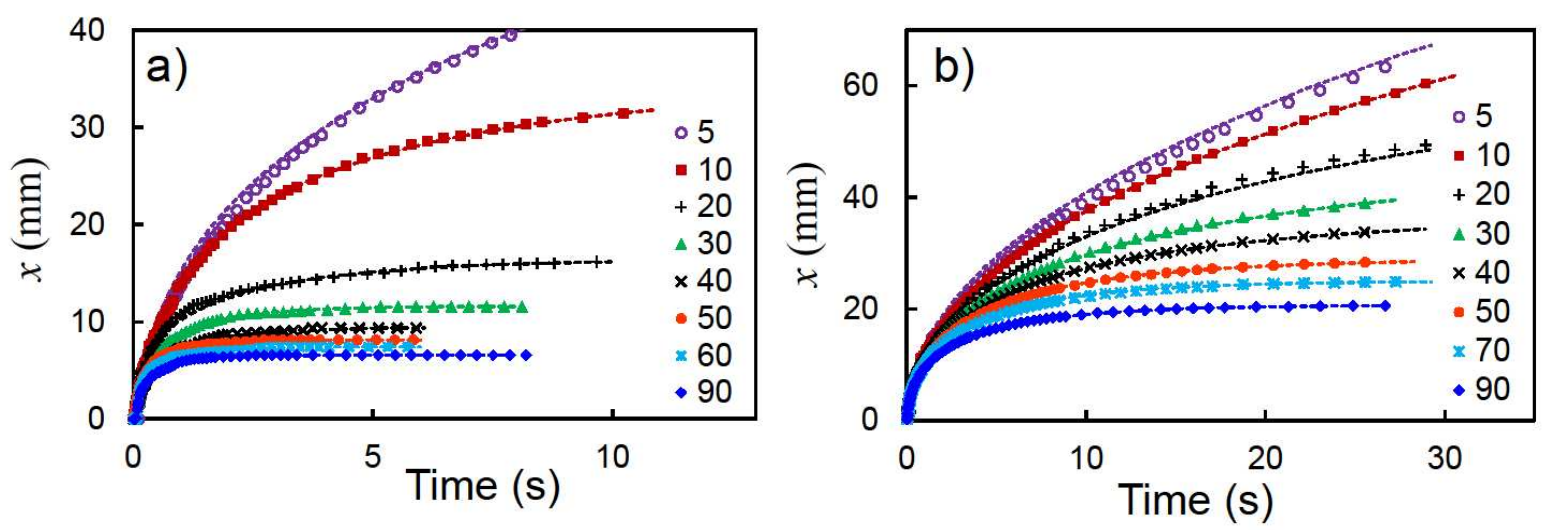

Figure 7: The effect of tilt angle, $\varphi$, on the dynamics of capillary penetration of 9.6 mPas PDMS oil in tubes with a) $R=650 \mu \mathrm{m}$ and b) $R=190 \mu \mathrm{m}$. The dashed lines are the corresponding viscogravitational fits.

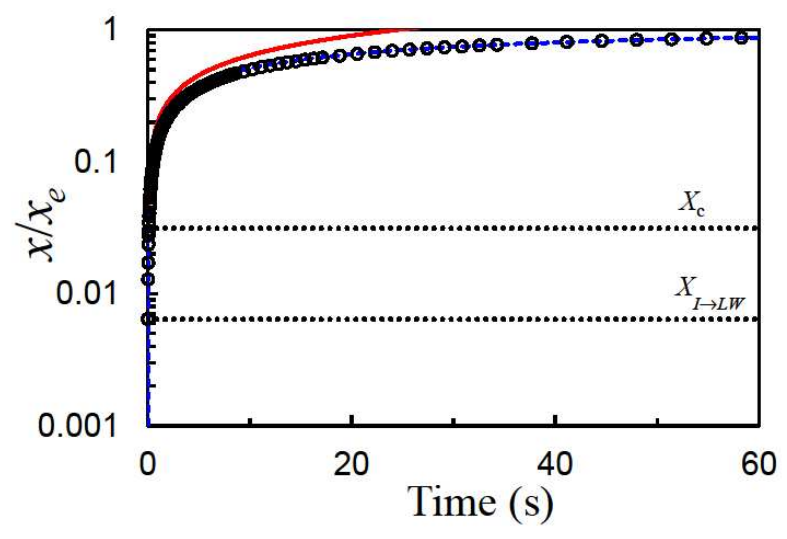

Figure 8. Capillary penetration of PDMS oil of viscosity $\eta=48 \mathrm{mPas}$ in a tube of radius $R=650 \mu \mathrm{m}$ at $\varphi=10^{\circ}$. The symbols represent the data points, and the solid and dashed lines represent the Bosanquet solution and visco-gravitational fits respectively. The dotted horizontal lines give cross-over lengths values $X_{I-L W}=0.006$ and $X_{\mathrm{c}}=0.031$. 


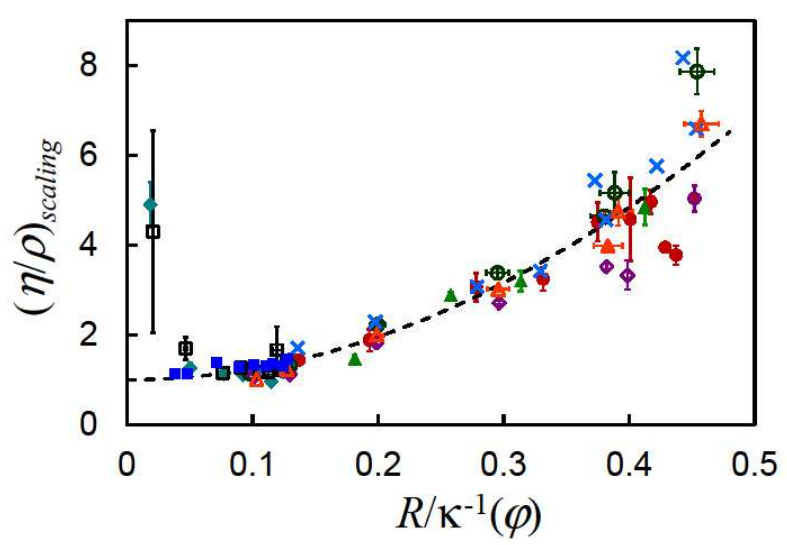

Figure 9: $\quad$ Dependence of the scaling factor $a_{\text {scaling }}=(\eta / \rho)_{\text {scaling }}$ on the effective $R / \kappa^{-1}(\varphi)\left(5^{\circ} \leq\right.$ $\left.\varphi \leq 90^{\circ}\right)$ in tilted tubes with radius $R=190 \mu \mathrm{m}(\diamond \diamond: \eta=9.6 \mathrm{mPas}, \square \square \square: \eta=19.2 \mathrm{mPas}, \mathbf{\square} \boldsymbol{\square} \eta=$ $48.0 \mathrm{mPas})$ and $R=650 \mu \mathrm{m}(\bullet \bullet \bullet: \eta=9.6 \mathrm{mPas}, \times \times \times: \eta=19.2 \mathrm{mPas}, \boldsymbol{\Delta} \boldsymbol{\Delta} \boldsymbol{\Delta}: \eta=48.0 \mathrm{mPas})$. Also shown is the data from fig. 2 in vertical tubes for $\eta=9.6 \mathrm{mPas}(\diamond \diamond \diamond), 19.2 \mathrm{mPas}($ ooo $)$ and $48.0 \mathrm{mPas}(\Delta \Delta \Delta)$. The dashed line is a guide to the eye for the general trend. 


\title{
Table of Contents Graphic
}

\begin{abstract}
"Capillary penetration into inclined circular glass tubes" by Christophe L. Trabi, F. Fouzia Ouali, Glen McHale, Haadi Javed, Robert H. Morris and Michael I. Newton.
\end{abstract}

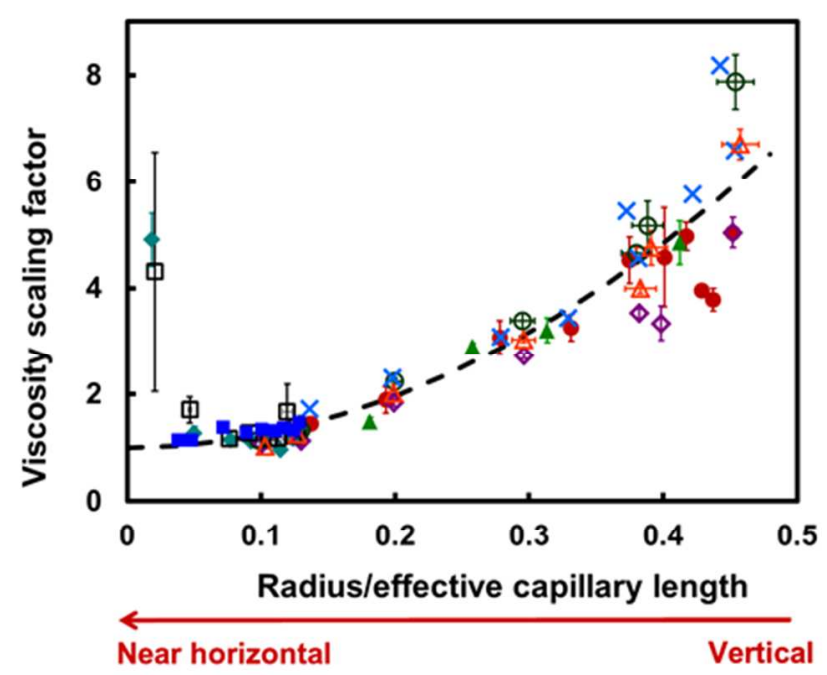

